PSI paper P1-028

\title{
Measurement of Transient Sputtering of Mo and W by Al and B Ions Injected by Laser Ablation in PISCES-A
}

\author{
E.M. Hollmann, D. Nishijima, and R.P. Doerner \\ Center for Energy Research, University of California-San Diego, La Jolla, CA 92093-0417, USA
}

\begin{abstract}
The development of a transient sputtering technique in PISCES-A plasmas is reported. A $\mathrm{Nd}$ :YAG laser is used to ablate impurities from a sample placed outside the plasma column, delivering a short $(\sim 10 \mu \mathrm{s})$ pulse of impurities into the plasma. The injected impurity ions travel down the plasma column and cause a brief $(\sim 10-100 \mu \mathrm{s})$ pulse of line emission from sputtered target material. This technique offers some advantages over steady-state sputtering experiments: a wide range of impurity ions can be injected, and incoming impurity ion and sputtered atom velocities can be inferred from time of flight measurements. Measurements of Mo and $\mathrm{W}$ sputtering by $\mathrm{Al}^{2+}$ and $\mathrm{B}^{+}$ions are presented. The Mo sputtering yields are found to be about 5-10 times lower than expected, consistent with steady state measurements of sputtering of $\mathrm{Mo}$ by $\mathrm{He}^{+}$under the same conditions. W sputtering is also lower than predicted.
\end{abstract}

PACS: 52.20.Hv, 52.25.Vv, 52.40.Hf

PSI-21 Keywords: Divertor material, erosion \& deposition, impurity seeding, plasma flow

Corresponding author address: Eric Hollmann, University of California-San Diego, 9500

Gilman Dr., La Jolla, CA, 92093-0417, USA

Corresponding/presenting author: Eric Hollmann

Corresponding/presenting author email: ehollmann@ucsd.edu 


\section{Introduction}

Understanding erosion of plasma-facing components is important for magnetic fusion research because surface erosion can affect core purity and performance, fuel codeposition and retention in the walls, and first wall lifetime [1]. While ion beam experiments can perform precision sputtering measurements [2], plasma sputtering experiments are also important. For example, changes in the surface structure (blisters [3], fuzz [4], etc) can affect erosion rates either via geometrical effects [5] or by changing surface binding energies [6]. Additionally, plasma effects on ion energies can affect sputtering yields, for example, by plasma transients [7], gyro-orbit effects [8], and flow entrainment [9].

Typically, material erosion experiments in plasmas are performed in quasi-steady state experiments. However, sputtering by transiently injected impurities into a steady-state plasma is potentially attractive because (a) almost any species can be injected, (b) the effect of net and gross erosion can be separated more easily (by looking for changes in steady state sputtering levels after the transient sputtering experiment is completed), and (c) energy information on the sputterer and sputtered material can be obtained from timeof-flight measurements.

Here, the technique of transient sputtering is demonstrated using laser ablation to inject a trace pulse of $\mathrm{Al}$ or $\mathrm{B}$ impurities into a cylinderical discharge of $\mathrm{He}^{+}$ions. Sputtering yields are found to be 5-10 times lower than predicted, similar to steady-state sputtering yields by He ions under the same plasma conditions. Attempts to validate the absolute 
calibration of the transiently injected impurity density are still ongoing, as are efforts to measure the angular distribution of transiently sputtered atoms.

\section{Experimental setup}

The experiments described here are performed in the PISCES-A linear reflex-arc device [10], shown in Fig. 1. Helium is used as a working gas, with fill pressure $P_{N} \approx 0.8 \mathrm{~Pa}$. A cylindrical, diameter $\phi=0.04 \mathrm{~m}$, plasma with electron density $n_{e} \approx 3 \times 10^{18} \mathrm{~m}^{-3}$ and electron temperature $T_{e} \approx 7 \mathrm{eV}$ is formed. The plasma flows down the axial magnetic field $B_{Z}=0.14 \mathrm{~T}$ and recycles on a target plate (temperature $800 \mathrm{C}$ ). Relative species concentrations (He-I, He-II, He-III) are roughly (10:1:0.01).

Impurity injection experiments are performed by ablating a side mounted sample with a pulse length $\Delta t \approx 6 \mathrm{~ns}$, energy $E \approx 220 \mathrm{~mJ} \mathrm{Nd}: \mathrm{YAG}(1064 \mathrm{~nm})$ laser pulse. The sample is mounted about $3 \mathrm{~cm}$ from the plasma edge inside a collimator with a diameter $\phi=1$ $\mathrm{cm}$, height $h=1 \mathrm{~cm}$ aperture in order to more axially localize the impurity deposition. The dominant species retained in the plasma column are found to be $B^{+}$for boron injection and $A l^{2+}$ for aluminum injection [11]. The perturbation to the target plasma is small from the injected impurities; this is confirmed by monitoring He neutral lines and plunging Langmuir probe ion saturation current $I_{\text {sat }}$ during impurity injection.

Fast injected and sputtered ion emission is measured by filterscopes (photomultiplier tube + interference filter combinations). Filterscope collection areas correspond roughly to the plasma diameter. Bandwidths of the interference filters are typically $5 \mathrm{~nm}$, which is 
sufficiently narrow to isolate the impurity lines from nearby He-I lines. Figure 2 shows gated CCD camera images at the middle window to illustrate the spatial localization of the injected impurities. Figure 2(a) shows an unfiltered image (dominated by He-I emission) with no impurity injection. Figure 2(b) shows an image of Al-II (466 nm) at time $t=1 \mu$ s (relative to the laser pulse time). Figures 2(c) and (d) then show later time steps $t=2$ and10 $\mu$ s, demonstrating impurity deposition into the plasma cylinder. For comparison, images of Al-I (396 nm) and Al-III (570 nm) are also shown. All images use a gate width of $1 \mu$ s with the exception of Fig. 2(f); here, due to the smaller level of Al-III emission, a gate width of $100 \mu$ s is used.

Ion temperatures in these experiments are sufficiently low that they are expected to give a small energy contribution relative to typical sheath potentials of $\sim 100 \mathrm{~V}$. The background $\mathrm{He}^{+}$ion temperatures are measured to be of order $\mathrm{T}_{i} \approx 1-2 \mathrm{eV}$ using a high-resolution spectrometer. Injected species ion temperatures less than $10 \mathrm{eV}$ are measured for both radial and axial views. Directed energies were measured to be less than $1 \mathrm{eV}$.

\section{Sputtering measurements}

Gated CCD images of Mo-I $550 \mathrm{~nm}$ emission from sputtered Mo are shown in Fig. 3. Figure 3(a) shows sputtering from the background He plasma with a diameter $\phi=3.75$ $\mathrm{cm}$ Ta target and $\phi=0.5 \mathrm{~cm}$ Mo button on center. Figure 3(b) shows He plasma sputtering on a $\phi=3.75 \mathrm{~cm}$ Mo sample. Figure 3(c) shows transient sputtering due to Al 
with a $-50 \mathrm{~V}$ target bias. Figure 3(d) shows similar data to Fig. 3(c) but with a $-150 \mathrm{~V}$ target bias.

Figure 4 shows normalized axial and vertical slices through the data of Fig. 3. It can be seen in Fig. 4(a) that the axial structure of Mo-I emission is slightly elongated for Al sputtering, suggesting a higher sputtered Mo neutral energy as compared with sputtering by He. Monte-Carlo simulations were used to estimate expected Mo-I brightness profiles. The simulations included electron impact but ignored ion and neutral collisions. A Thompson energy distribution was assumed and angular distributions were assumed to be either diffusive $(\cos (\theta))$ or butterfly [12]. It can be seen that the measured axial profiles are slightly broader than predicted from the simulations.

Radial profiles are shown in Fig. 4(b); it can be seen that all the measured radial profiles are fairly similar. This contrasts with the expected radial profile for the data with the small $(\phi=0.5 \mathrm{~cm})$ Mo button, suggesting that Mo from the central button has migrated over onto the rest of the sample. This hypothesis is supported by post-exposure electron dispersive $\mathrm{x}$-ray analysis (EDS) giving roughly $50 / 50 \mathrm{Mo} / \mathrm{Ta}$ on the button and 4/96 $\mathrm{Mo} / \mathrm{Ta}$ on the tantalum body. Because of the $1 \mu \mathrm{m}$ analysis depth, this does not reveal the actual near-surface concentrations, but nevertheless clearly demonstrates that crosssample material migration is occuring here.

Fig. 5(a)-(c) show Al-III density vs time at different windows. This data is obtained from filterscopes, together with the background plasma $n_{e}, T_{e}$ profiles at each location and the 
Al-III photon emissision coefficients predicted by ADAS [13]. The data is then fit by a convective-diffusive model to estimate the Al-III flux to the target [11]. This model uses the middle window data as a source term, then varies a constant axial impurity diffusion coefficient, convective velocity, and radial loss rate to best match the target and source window data. With the collimator used here, two distinct peaks are often observed: the first peak is associated with direct impurity deposition by the laser produced plasma, while the second is associated with secondary processes like self-sputtering and arcing. This is supported by the observation that the second (but not the first) peak can be increased by applying a large (e.g. $-250 \mathrm{~V}$ ) reverse bias to the side sample. Figure 5(d) shows the resulting estimate for the aluminum flux to the target. The Mo flux leaving the target is estimated from filterscope data of Mo-I $(550 \mathrm{~nm})$ taken in front of the target sample, together with the local background plasma parameters and previously measured values of the Mo-I (550 nm) photon efficiency, S/XB [14].

An estimate of the sputtering yield $Y$ is obtained from the data of Fig. 5(d) by summing the total Mo flux out of the target and dividing by the total Al flux into the target. At this point, we do not attempt to separate sputtering yields from the first and second peaks. Also, redeposition of Mo is ignored, as is deposition of Al. The absence of significant Al deposition is supported by the observation that the background Mo-I signal between laser pulses remains, on average, constant. Typically, 5 - 10 laser pulses at $1 \mathrm{~Hz}$ are used to allow averaging and improve statistics. 
Figure 6 gives an overview of sputtering yields obtained with different sample materials. In each case, the ion incident energy is varied by varying the target bias. Incident energy is then assumed to be the ion charge ( +1 for $\mathrm{He}$ and $\mathrm{B}$ and +2 for $\mathrm{Al})$ times the sheath potential $-\left(V_{p}-V_{\text {bias }}\right)$ where $V_{\text {bias }}$ is the target bias and $V_{p} \approx-10 \mathrm{~V}$ is the plasma potential. The effects of injected ion energy and impurity ion flow entrainment are expected to be small $(<10 \mathrm{eV})$ in these experiments and are not included. Figure 6(a) shows the steady sputtering yield of the background helium plasma on Mo. The flux of He ions to the target sample is obtained from the measured downstream electron density profile and the spectroscopically measured helium ion parallel flow speed. Figure 6(b) shows $Y$ for transient sputtering of Mo by Al. Figure 6(c) shows $Y$ for transient sputtering of Mo by B and Figure 6(d) shows Y for transient sputtering of W by Al. In each case, TRIM simulations of sputtering yields are shown. For comparisons with Al data, sputtering rate simulations for $\mathrm{Ne}$ and Ar projectiles are shown, since these are already published and are expected to be close to Al sputtering rates [15]; similarly, existing C sputtering rate simulations are used as an approximation for sputtering by B. For Al data, sputtering rates due to Ne and Ar projectiles are shown, since these are already published and are expected to bracket Al sputtering rates [15]. Analysis in each case is similar to that shown in Fig. 5. For B injection, B-II $(345 \mathrm{~nm})$ is used to track the dominant B species. For W sputtering, the $429.4 \mathrm{~nm}$ W-I line is used, together with previously measured $\mathrm{S} / \mathrm{XB}$ values [16]. Because of the unkown extent of material migration in experiments with sample size $\phi<3.8 \mathrm{~cm}$, it is simply assumed that $\phi=3.8 \mathrm{~cm}$ for analyzing $Y$, i.e. we assume that the sample material (Mo or $\mathrm{W}$ ) has migrated to cover the whole sample cap. This approximation is probably somewhat valid in the $\phi=0.5 \mathrm{~cm}$ data 
of Fig. 6(a) and 6(b), where the small Mo button is held by a Ta cap which is more difficult to sputter. The approximation may not be valid however in the data of Fig. 6(d), where $\mathrm{W}$ is held by a Ta cap. The reason for the larger difference between large and small target samples in the He sputtering case, Fig. 6(a), compared with the Al sputtering case, Fig. 6(b), is not understood at present. It is possible that the slower He sputtering is not giving the assumed full Mo migration over the full sample cap over the experiment time scale. All Mo sputtering yields are scaled by a factor of 1.7 to account for radial loss of neutrals out of the observation volume predicted by the Monte-Carlo simulations. This geometrical correction factor is omitted for the W data of Fig. 6(d). The Monte-Carlo simulations were not run for the $\mathrm{W}$ data case, as this is geometrical correction expected to give a perhaps $50 \%$ correction, while the uncertaintly in actual sample area is larger, of order a factor 3 .

\section{Conclusions}

The technique of sputtering with transient impurity deposition using laser ablation has been demonstrated successfully. This method has the advantage over standard steadystate plasma sputtering measurements in that sputtering due to almost any material can be measured. Additionally, the dynamics of the injected impurity and the sputtered material can be measured with time-of-flight measurements, in principle providing information on the energy distribution of both. The disadvantages of the transient sputtering technique are that it is more difficult to calibrate than steady sputtering (which can use sample weight loss). The PISCES-A plasmas have fluctuation levels in $n_{e}$ of order unity, typically requiring averaging over $5-10$ shots to obtain good transient sputtering data. 
After this averaging, fluctuation levels in filterscope signals from plasma oscillations only give fairly small errors of order $10 \%$. However, the errors in the atomics physics calculations (ADAS photon emission coefficients) when compared with weight loss measurements in steady-state sputtering experiments can frequently be a factor of 2-3. The dominant error in the sputtering yield data of Fig. 6 is therefore this photon emission coefficient error of order 2-3. Efforts were made to calibrate the injected impurity density by looking for a transient $n_{e}$ perturbation during probe plunges or from He-I signals; however, these efforts have not been successful yet. Future work will evaluate calibration of the transient sputtering using targets briefly exposed at zero bias (low sputtering) and measuring resulting thin film deposition.

Another challenge was found to be material migration - small sample buttons were used to attempt to reduce radial loss of sputtered neutrals and also to study the angular distribution of sputtered neutrals; however, these experiments found that a mixture of species quickly resulted. The most reliable new results presented here are therefore the transient sputtering measurements with the single species $\phi=3.8 \mathrm{~cm}$ Mo sample. These experiments obtain a sputtering yield of order 5-10 times smaller than predicted by TRIM, similar to what is observed with steady-state He sputtering. Previously, this decreased sputtering yield was hypothesized to be due to He residing near the surface, which could absorb incoming atom momentum [17].

Another future goal of this work is the observation of enhanced sputtering due to flow entrainement. Heavier species can become entrained in the parallel flow of a lighter 
background plasma, giving significantly higher effective directed energies and increasing sputtering yields [9]. In these experiments, the effect is expected to be of order $5-10 \mathrm{eV}$ and therefore improved data quality will be necessary to verify the presence of this effect.

\section{Acknowledgements}

This work was supported by U.S. DOE grant DE-FG02-07ER54912. The assistance of M. Baldwin, L. Chousal, R. Hernandez, A. Pigarov, and T. Lynch is gratefully acknowledged. The originating developer of ADAS is the JET Joint Undertaking.

\section{References}

[1] G. Federici, C.H. Skinner, J.N. Brooks, et al., Nucl. Fusion 41, 1967 (2001).

[2] D. Fink and L.T. Chadderton, Rad. Effects and Defects in Solids, 160, 67 (2005).

[3] M. Balden, V. Rohde, S. Lindig, et al., J. Nucl. Mater. 438, S220 (2013).

[4] M. Baldwin and R.P. Doerner, J. Nucl. Mater. 404, 165 (2010).

[5] R.P. Doerner, D. Nishijima, and T. Schwarz-Selinger, Phys. Scr. T159, 014040 (2014).

[6] K. Schmid, M. Baldwin, and R.P. Doerner, J. Nucl. Mater. 348, 294 (2006).

[7] A. Jarvinen, M. Groth, D. Moulton, et al., J. Nucl. Mater. 438, S1005 (2013).

[8] A.V. Chankin, D.P. Coster, and R. Dux, Plasma Phys. Contr. Fusion 56, 025003 (2014).

[9] P. L. Stangeby, J. Phys. D 20, 1472 (1987). 
[10] D. M. Goebel, G. Campbell, and R. W. Conn, J. Nucl. Mater. 121, 27 (1984).

[11] E.M. Hollmann, K.R. Umstadter, R.P. Doerner, et al, J. Nucl. Mater. 415, S425 (2011).

[12] E. Oyarzabal, J.H. Yu, R.P. Doerner, and G.R. Tynan, J. Appl. Physics 100, 063301 (2006).

[13] H. P. Summers, W. J. Dickson, M. G. O’Mullane, et al., Plasma Phys. Control. Fusion 48, 263 (2006).

[14] D. Nishijima, R.P. Doerner, D.G. Whyte, et al., J. Phys. B 43, 225701 (2010).

[15] W. Eckstein, “Calculated sputtering, reflection, and range values,” IPP 9/132 (MPG Garching, 2002).

[16] D. Nishijima, R.P. Doerner, M.J. Baldwin, et al, Phys. Plasmas 18, 019901 (2011).

[17] R.P. Doerner, C. Bjorkas, D. Nishijima, and T. Schwarz-Selinger, J. Nucl. Mater. 438, S272 (2013). 


\section{Figure captions}

Fig. 1. Experimental schematic showing side sample used to create laser plasma and target sample used to study sputtering by impurities entrained in the plasma flow.

Fig. 2. Middle window port visible images showing (a) background plasma emission, (b) $t=1-2 \mu \mathrm{s} \mathrm{Al-II,} \mathrm{(c)} t=2-3 \mu \mathrm{s} \mathrm{Al-II,} \mathrm{(d)} t=10-11 \mu \mathrm{s} \mathrm{Al-II,} \mathrm{(e)} t=10-11 \mu \mathrm{s} \mathrm{Al}-\mathrm{I}$, and (f) $t$ $=2-100 \mu \mathrm{s}$ Al-III (with peak emission around $t \sim 10 \mu \mathrm{s}$ )

Fig 3. Gated CCD images of Mo-I sputtered by (a) and (b) He and (c) and (d) Al. (b) uses a $\phi=3.75 \mathrm{~cm}$ Mo target, while (a), (c), and (d) use a $\phi=3.75 \mathrm{~cm}$ Ta target with a $\phi=0.5$ cm Mo button on center. (a) and (b) use $10 \mathrm{~ms}$ integration time, while (c) and (d) use 300 $\mu$ s integration time.

Fig 4. (a) axial and (b) vertical profiles of Mo-I brightness measured for same cases as shown in Fig. 3.

Fig. 5. Time sequences of injected Al and sputtered Mo showing (a) Al-III density in middle window, (b) Al-III density in target window, (c) Al-III density source window, and (d) flux of Al to target and Mo from target.

Fig. 6. Sputtering yields measured for (a) steady sputtering of Mo by He, (b) transient sputtering of Mo by Al, (c) transient sputtering of Mo by B, and (d) transient sputtering of $\mathrm{W}$ by Al. 


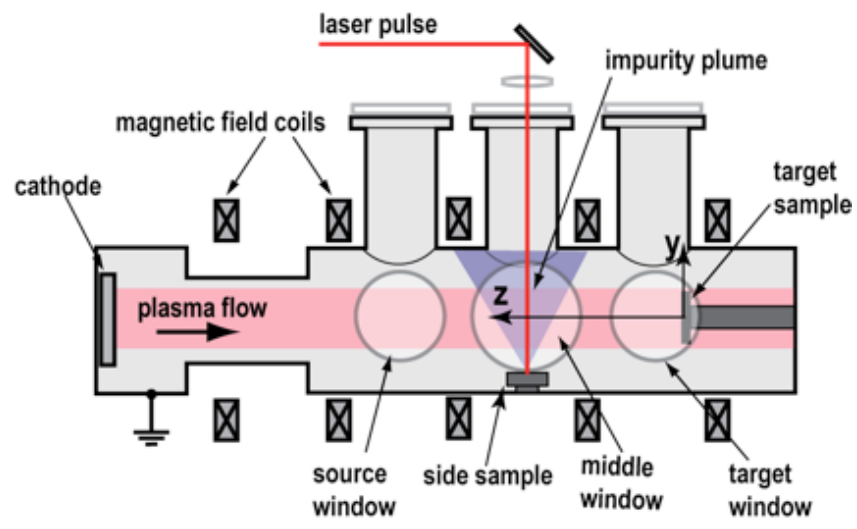

Figure 1 

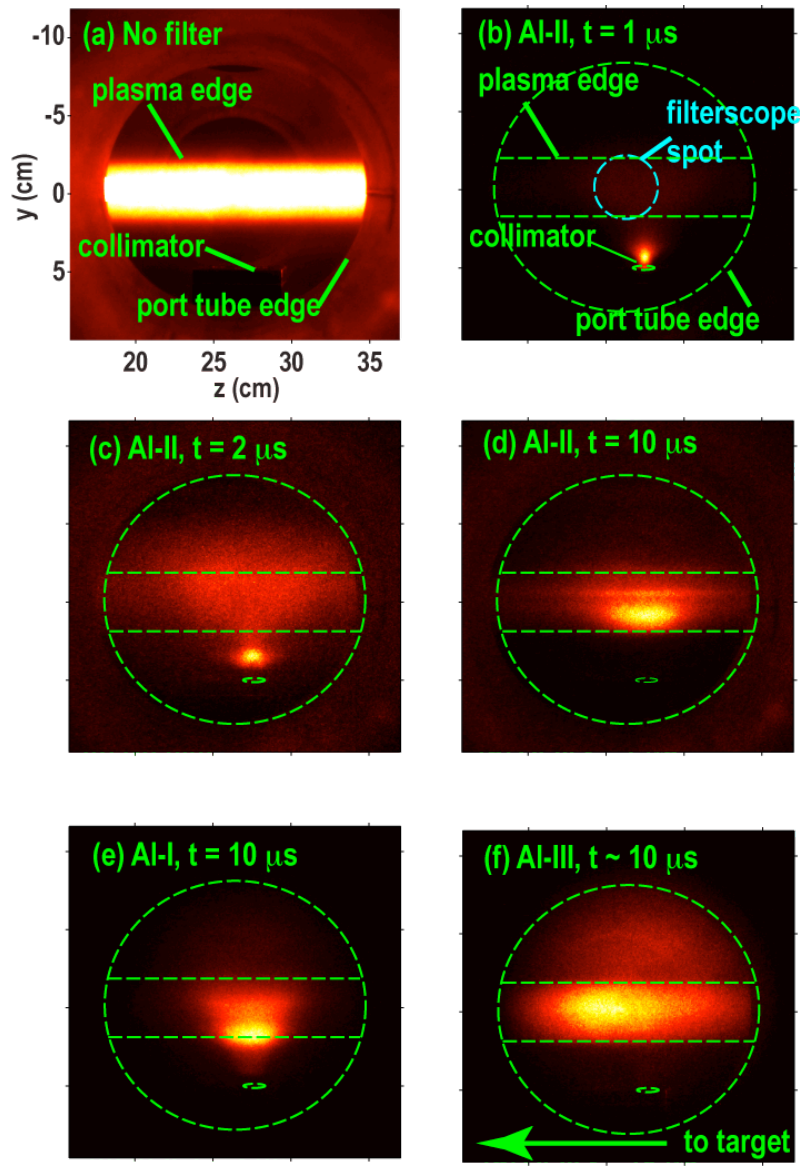

Figure 2 


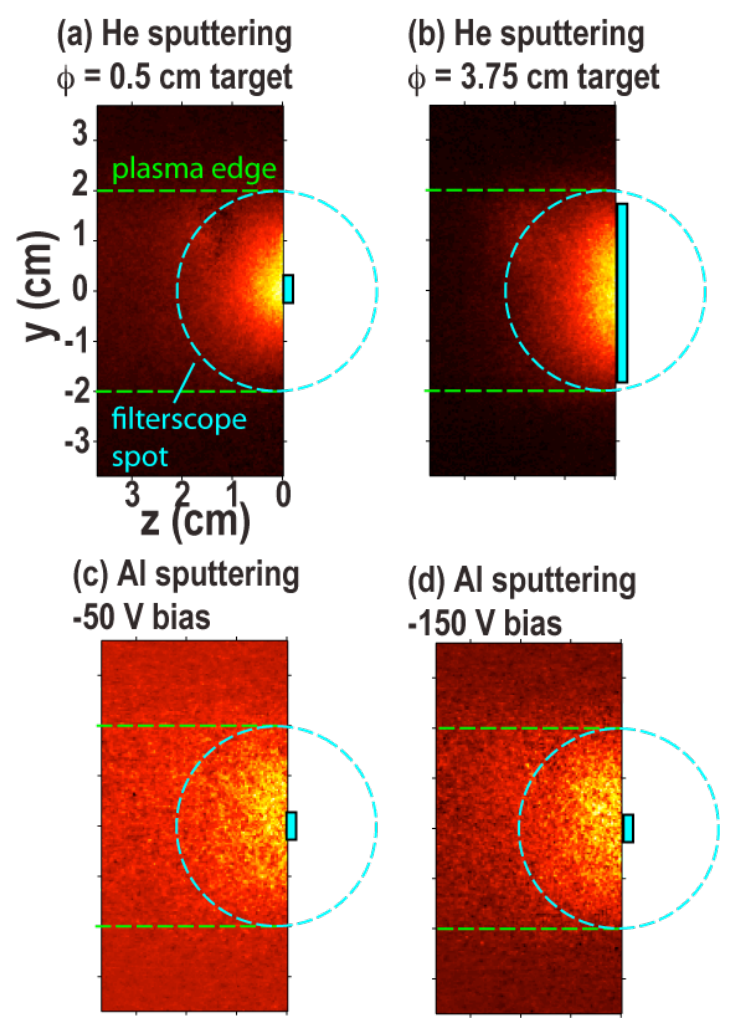

Figure 3 


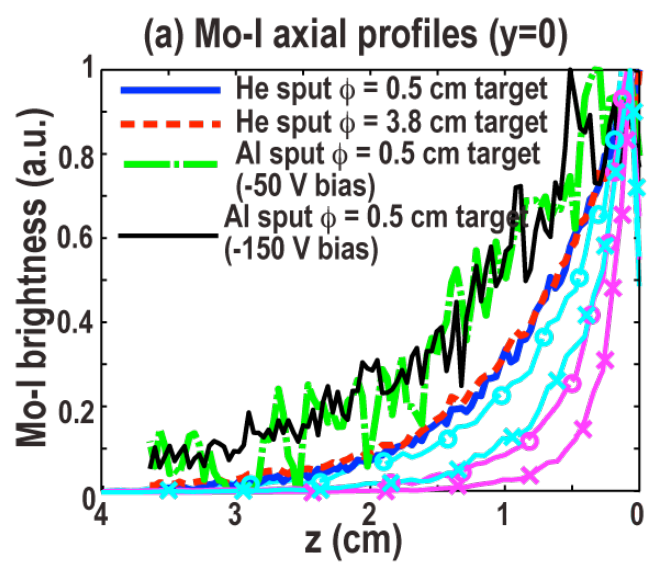

(b) Mo-I vertical profiles $(z=0)$

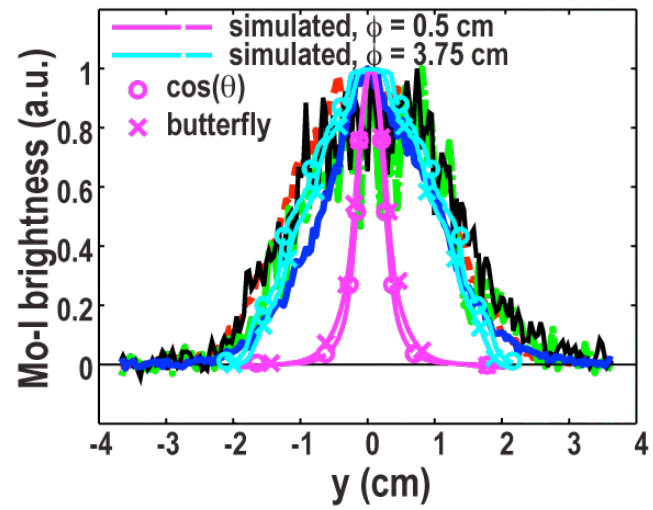

Figure 4 

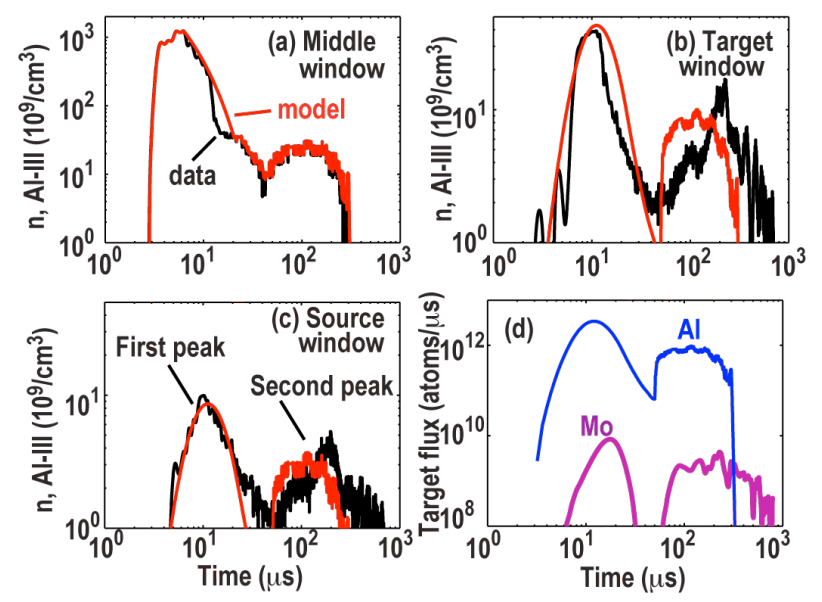

Figure 5 

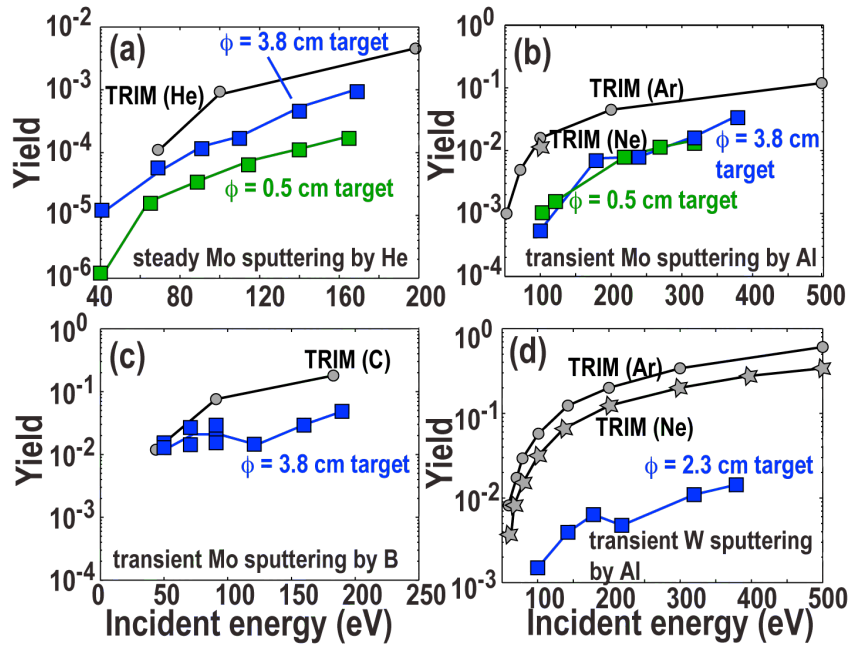

Figure 6 

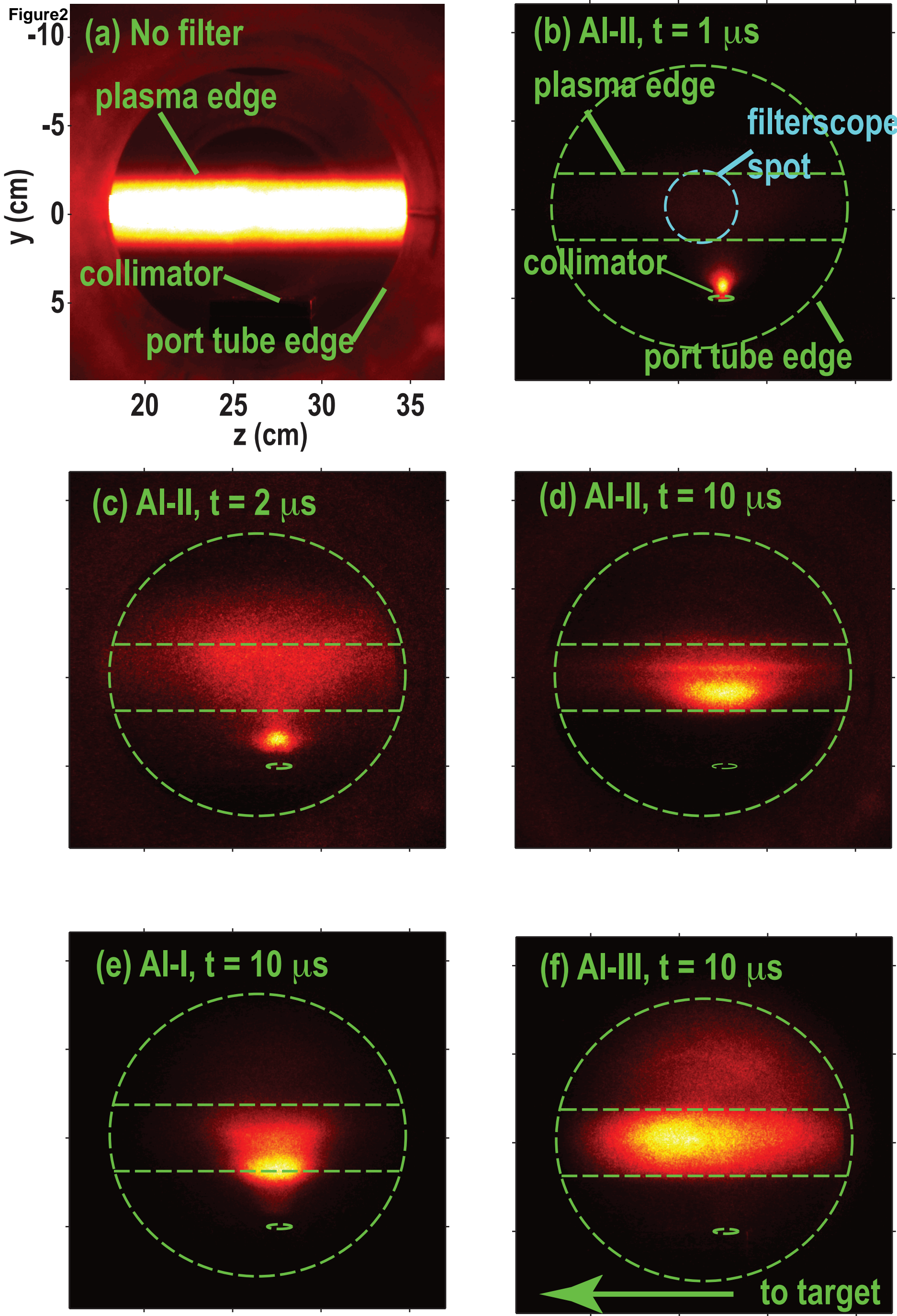
Figure4

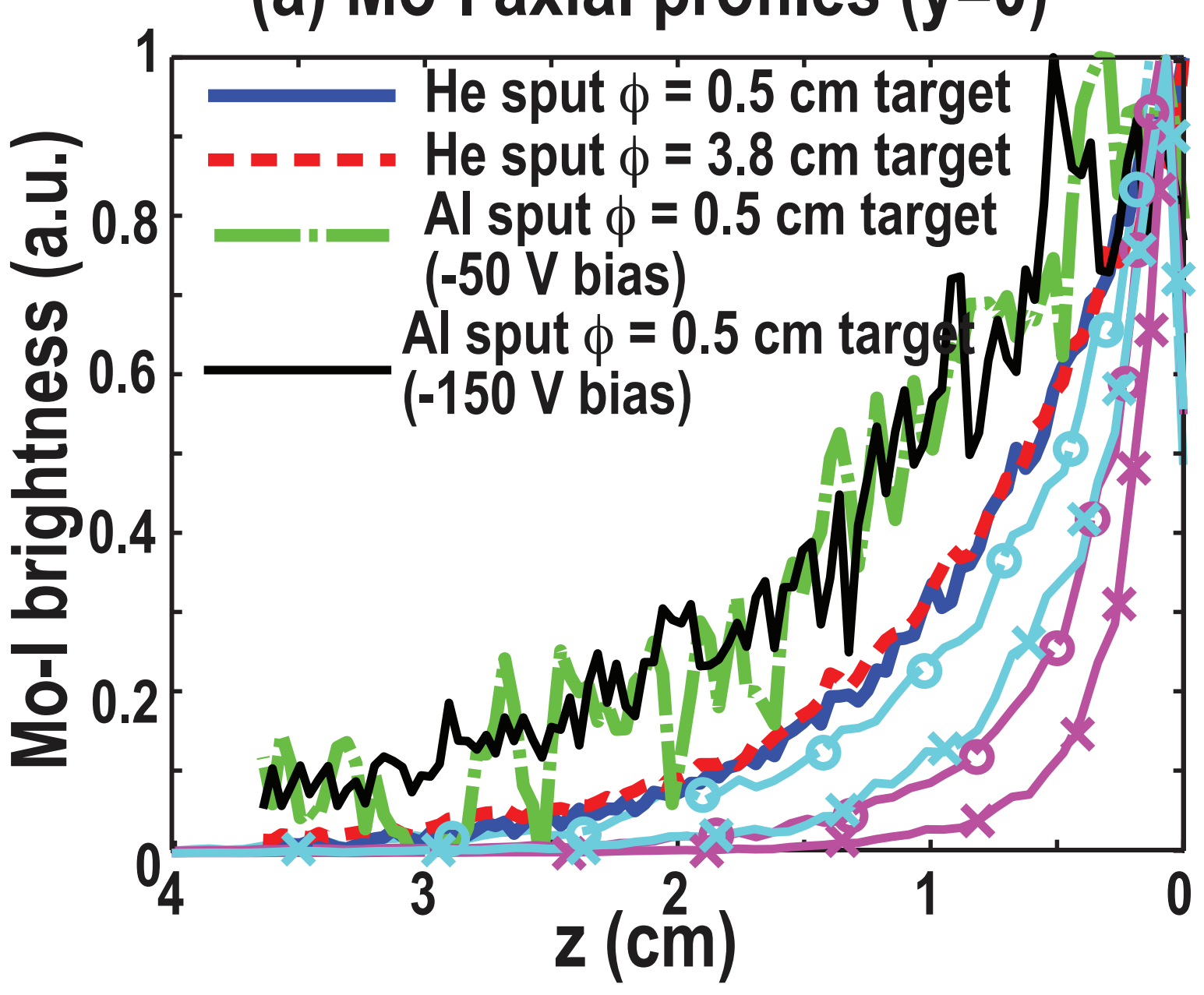

(b) Mo-I vertical profiles $(z=0)$

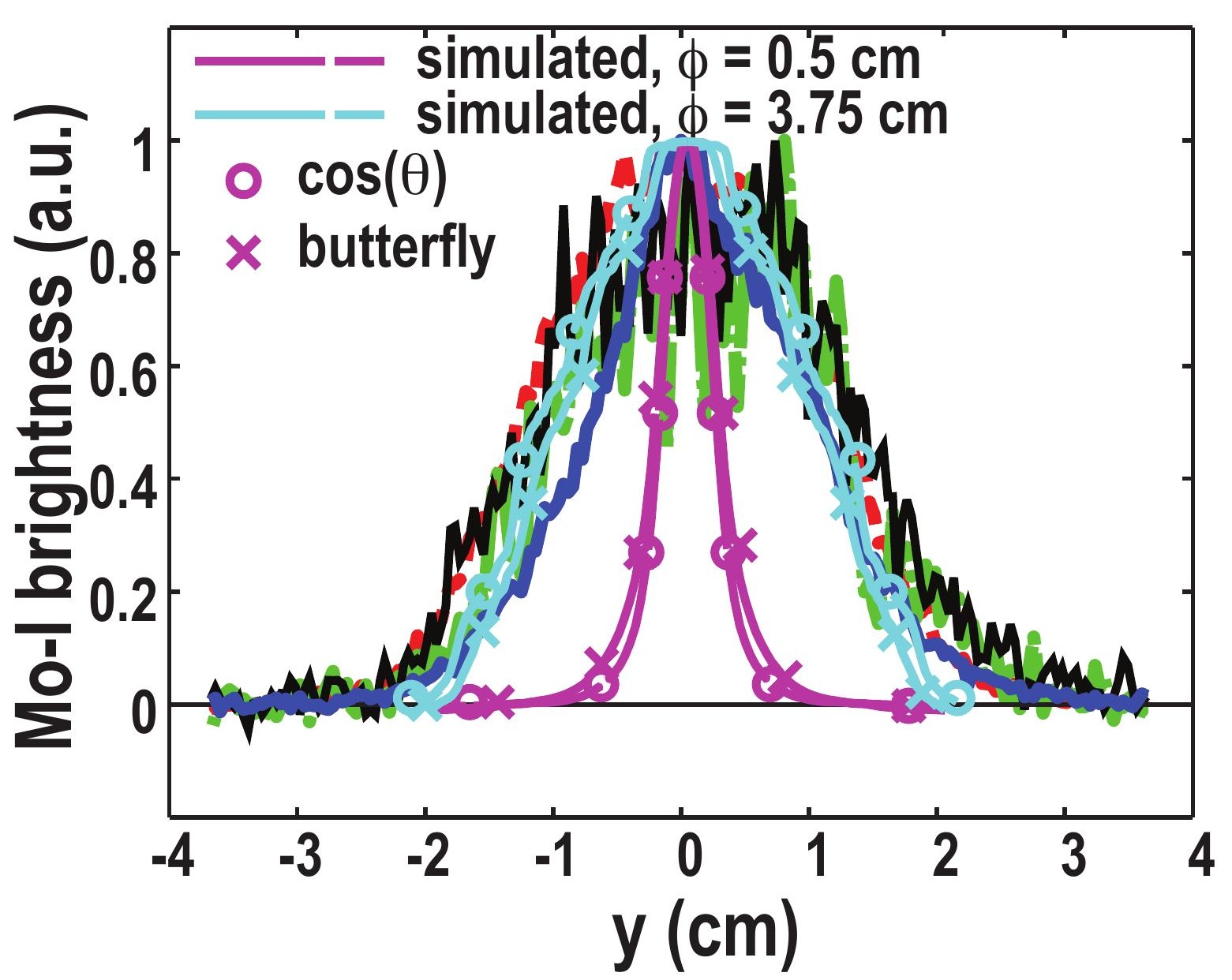




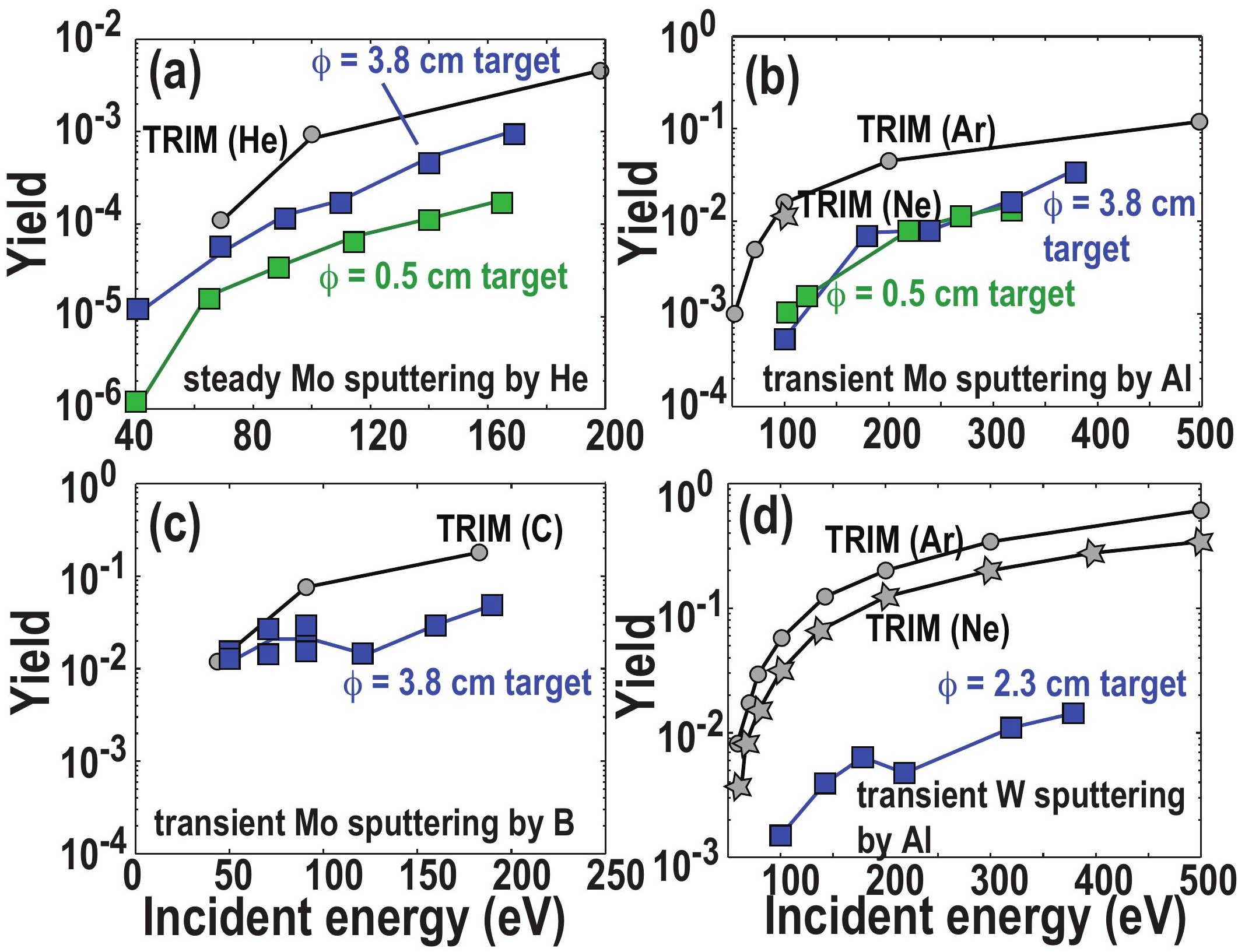

